\title{
Differential platelet oxidation-inflammatory phenotype in coronary artery disease versus peripheral artery disease supports diverse pathophysiological processes
}

\section{Kanika Jain}

Yale University

Tarun Tyagi

Yale University

John Hwa

Yale University

Costin lonescu ( $\sim$ cOSTIN.IONESCU@YALE.EDU)

Yale University

\section{Research Article}

Keywords: platelets, oxidative stress, antiplatelet therapy.

Posted Date: December 21st, 2020

DOl: https://doi.org/10.21203/rs.3.rs-128004/v1

License: (c) (1) This work is licensed under a Creative Commons Attribution 4.0 International License.

Read Full License 
Differential platelet oxidation-inflammatory phenotype in coronary artery disease versus peripheral artery disease supports diverse pathophysiological processes

Kanika Jain $\mathrm{PhD}^{1}$, Tarun Tyagi PhD${ }^{1}$, John Hwa MD PhD¹, Costin N. Ionescu MD PhD²*

${ }^{1}$ Yale Cardiovascular Research Center, Section of Cardiovascular Medicine, Department of Internal Medicine, Yale University School of Medicine, 300 George St Level 7, New Haven CT 06511

${ }^{2}$ Yale Cardiovascular Medicine, Department of Internal Medicine, Yale-New Haven Hospital St Raphael Campus, 1450 Chapel St, New Haven CT, 06511

* Corresponding author: Costin lonescu MD PhD, Yale Cardiovascular Medicine, Yale-New Haven Hospital St Raphael Campus, 1450 Chapel St, New Haven CT, 06511, costin.ionescu@yale.edu.

Brief Title: Platelet redox homeostasis in CAD vs PAD

Keywords: platelets, oxidative stress, antiplatelet therapy. 


\section{ABSTRACT}

Platelets are major contributors to atherothrombosis. There is emerging evidence that atherothrombosis pathogenesis may be different between vascular beds. In a prospective casecontrol study, we compared platelet oxidation-inflammatory status from patients with coronary artery disease CAD, peripheral artery disease (PAD) and a young healthy cohort. All patients had stable disease with no recent acute CAD or PAD events. Assessment of 75 eligible patients revealed a typical outpatient cardiology cohort. A significant increase in platelet activation (soluble P-selectin and CD4OL) was seen in CAD. Consistent with this platelet activation, the CAD group had the highest levels of oxidants (reactive oxygen species, lipid peroxidation and protein carbonylation) and the lowest levels of antioxidants (catalase and superoxide dismutase enzyme activity). IL1 $\beta$ was significantly increased in the PAD group. The significant differences in the oxidation-inflammatory parameters, between patients with CAD versus PAD, may explain different atherothrombosis pathology, as well differential response to therapies, such as aspirin and P2Y12 inhibitors. Whether these are the cause or consequence of disease or both, requires further extensive exploration. Such mechanistic understanding is important in developing disease targeted therapies. 


\section{INTRODUCTION}

Heart disease continues to be the leading cause of death in the United States[1]. The primary cause of heart disease is CAD, a chronic immune-inflammatory disease affecting the coronary arteries [2]. Formation of atheroma, the central manifestation of atherosclerosis, requires the interplay of multiple cell types and mediators [3]. There is growing evidence supporting an essential role for platelets and reactive oxygen species (ROS) in the development of atherosclerosis [4]. Activated platelets are involved in the initial atheroma formation and also in the progression of existing atheromatous lesions $[5,6]$.

Peripheral vascular disease (PAD) (usually referring to arterial disease of the lower extremities) is considered a similar atherosclerotic process to CAD but in a different vascular bed. However, there are emerging major clinical differences between CAD and PAD patients. In the international REACH registry, PAD patients had higher cardiovascular (CV) death and major CV events than CAD or cerebrovascular disease (CVD) patients [7]. As opposed to CAD, PAD patients outcomes are improved by therapy with $\mathrm{P} 2 \mathrm{Y} 12$ inhibitors compared to aspirin [8] and anticoagulation therapy [9]. In addition, platelets from patient with PAD appear to react differently to shear stress [10], have different platelet reactivity [11] and respond differently to angioplasty [12]. Thus, the pathophysiological role played by platelets in PAD versus CAD may be quite different in regard to the atherosclerotic pathology.

The difference in outcomes between CAD and PAD patients may be related to distinct platelet biology. A phenotypical different platelet population was recently proposed as an alternative explanation for the different outcomes in patients with acute non-ST vs ST-segment elevation myocardial infarction [13]. It is believed that chronic atherothrombotic disease of the coronary and peripheral arteries are associated with similar platelet phenotypes. We now conduct an exploratory analysis to evaluate potential differences between platelets obtain from patients with chronic CAD and PAD (no recent acute events). We report that platelets from patients with stable CAD and PAD have different oxidative stress phenotypes. Platelets from CAD patients have high ROS levels associated with lower anti-oxidant enzyme activity. In contrast, platelets from 
PAD patients have low normal levels of ROS and anti-oxidant enzyme activity but increased plasma IL1 $\beta$. These observations may in part explain the differential effects of antiplatelet agents on PAD versus CAD and the phenotypic differences.

\section{METHODS}

The study protocol and assays have been previously described and reported by our group [14].

\section{Patient recruitment}

310 consecutive patients presenting to an outpatient cardiovascular clinic were screened for inclusion in the study (Figure 1).

Figure 1. Schematic Overview of the study. Flow diagram outlining the patient screening and enrollment.

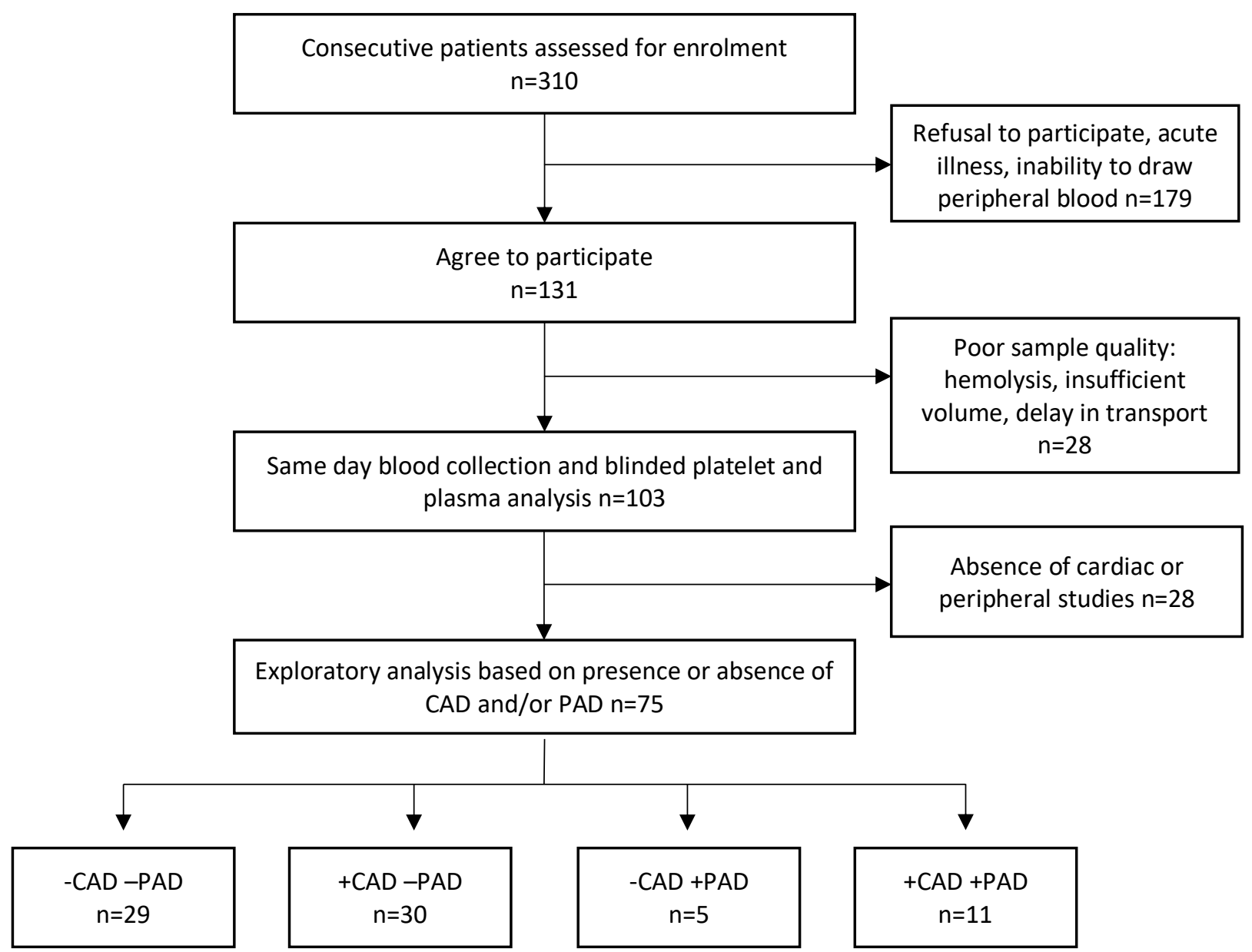


A total of 179 patients were excluded due to consent issues, recent/current acute illness, or issues with blood draw. As fresh platelets are needed for accurate oxidative stress measurements, 28 patients were excluded due to poor sample quality, blood hemolysis, insufficient volume, or delay in transport. For the present analysis only patients with prior cardiac investigations (stress test and/or coronary angiography) to determine the presence or absence of CAD were included $(n=75)$. Patients were considered to have CAD if there was a positive stress test or any visual coronary artery stenosis on coronary angiography. PAD was defined by the presence of claudication, or an abnormal ankle brachial index, arterial ultrasound or peripheral angiography study. Patients were divided into 5 groups: healthy young controls with no known medical conditions, patients with no CAD or PAD (-CAD-PAD), patients with CAD but no PAD (+CAD), patients with no CAD but PAD (+PAD), and patients with both CAD and PAD (+CAD+PAD). Although overall numbers were small, there are three important features for this cohort 1) they reflect real world numbers where the proportion of outpatients with clinically diagnosed +PAD are small compared to $+C A D$ or $-C A D-P A D ; 2$ ) the characterization of platelet oxidative state is amongst the most detailed of any platelet publication; and 3) all the assays were performed blinded. These studies were approved by the Yale Human Investigation Committee (\#1005006865). Informed consent was obtained from each subject and conform to the principles set out in the WMA Declaration of Helsinki and the Belmont Report.

\section{Isolation of human platelets}

As previously described [14], venous blood was drawn from patients or healthy adult volunteers and platelet-rich plasma (PRP) was prepared by centrifugation at $220 \mathrm{~g}$ for $12 \mathrm{~min}$ at $25^{\circ} \mathrm{C}$. WBC exclusion was confirmed by viewing under phase contrast microscope. Washed platelets were prepared by centrifugation of PRP (upper $2 / 3$ of supernatant) at $700 \mathrm{~g}$ for $8 \mathrm{~min}$. PGI2 (20 ng/ml) and apyrase were added to the PRP. Platelet pellet thus obtained was gently resuspended in calcium free Platelet washing buffer $(103 \mathrm{mmol} / \mathrm{l} \mathrm{NaCl}, 5 \mathrm{mmol} / \mathrm{l} \mathrm{KCl}, 1 \mathrm{mmol} / \mathrm{l} \mathrm{MgCl} 2,5 \mathrm{mmol} / \mathrm{l}$ glucose, $36 \mathrm{mmol} / \mathrm{I}$ citric acid, $0.35 \% \mathrm{BSA}, \mathrm{pH} 6.5$ ). This step was repeated to obtain washed platelets needed for the biochemical assays, and flow cytometry-based assays. 


\section{ELISA}

Soluble platelet activation markers were assayed by commercially available enzyme immunoassays specific for soluble P-selectin and soluble CD40L (Affymetrix), IL-1, IL-6 and TNF$\alpha$ (Invitrogen) as per the manufacturer's guidelines.

\section{Detection of intracellular ROS}

Levels of platelet-derived intracellular $\mathrm{H}_{2} \mathrm{O}_{2}$ were measured using $2^{\prime}, 7^{\prime}$ dichlorodihydrofluorescein diacetate (H2DCF-DA; Molecular Probes). Washed platelets were incubated H2DCF-DA and the fluorescence intensity was detected by a microplate reader (Biotek) using excitation at $492 \mathrm{~nm}$ and emission at $517 \mathrm{~nm}$ wavelengths. DCFH-DA is cell permeable and diffuses across the platelet cytomembrane. It is hydrolyzed in platelet cytoplasm to form nonfluorescent DCFH, which loses the ability to diffuse across the cytomembrane. ROS rapidly induces DCFH and undergoes one-electron oxidation to generate DCF, which is a fluorescent compound. Therefore, the reactive oxygen species production is reflected by an increase of DCF radicals in platelets. Results are expressed as mean fluorescence intensity.

\section{Protein carbonylation assay}

Protein carbonyl content is the most common indicator of protein oxidation. The levels of protein carbonyl in platelets were determined using a Protein carbonyl content Assay Kit (Abcam, US) and all the procedures were performed as per the manufacturer's instruction. Results are expressed as nmol carbonyl/mg protein.

\section{Catalase activity}

The catalase (CAT) activity was assayed using the catalase colorimetric activity kit (Invitrogen, Life Technologies). Briefly the platelet lysate was diluted 1:10 in the Assay Buffer provided and assayed as per the manufacturer's instructions. One unit of catalase $(U)$ decomposes $1.0 \mu \mathrm{mol}$ of $\mathrm{H}_{2} \mathrm{O}_{2}$ per min at $\mathrm{pH} 7.0$ and $25^{\circ} \mathrm{C}$. The results are expressed as $\mathrm{U} / \mathrm{mg}$ protein. 


\section{SOD activity}

Superoxide Dismutase (SOD) activity was measured using the SOD Colorimetric Activity kit (Invitrogen, Life Technologies). Briefly the platelet lysate was diluted 1:5 in the Assay Buffer provided and the activity assayed as per the manufacturer's instructions. One Unit (U) of SOD is the amount of enzyme causing half the maximum inhibition of $1.5 \mathrm{mM}$ nitroblue tetrazolium reduction in the presence of riboflavin at $\mathrm{pH} 7.8$ and $25^{\circ} \mathrm{C}$. The results were expressed as $\mathrm{U} / \mathrm{mg}$ protein.

\section{Statistical analysis}

Given the interpatient variability when assessing oxidation parameters there were four key components to our analysis; 1 ) multiple assays were performed to support the same conclusions (e.g. ROS levels, lipid peroxidation, protein carbonylation); 2) comparison to a young healthy cohort was key in demonstrating the anticipated significant differences with aging; 3) assessing consistent changes in mean levels despite not being statistically different; and 4) using correlation to assess individual differences in multiple parameters.

Values are presented as mean \pm standard error of the mean (SEM). The sample size or experiments used for each study is specified for each figure legend. Differences between group means were assessed using the non-parametric $t$-test for continuous variables and $\chi 2$ or Fischer exact tests for categorical variables where appropriate. Two-sided $P<.05$ was considered statistically significant. Comparisons between two samples were performed using the Student's t-test. Data sets with three or more groups were analyzed by one-way ANOVA with a Bonferroni post hoc test. Analysis was performed with Prism 8.0 software (GraphPad Software, Inc., La Jolla, CA). $P$ values $<0.05$ were considered significant.

\section{RESULTS}

Of the 75 patients that met the inclusion/exclusion criteria and had a full set of data it was not surprising that only five (7\%) had +PAD (without clinical evidence of significant CAD) compared to $30(40 \%)$ that had +CAD (without clinical evidence of significant PAD) (Table 1). 
Table 1. Baseline clinical characteristics of patients stratified according to the underling atherosclerotic disease. $P$ values calculated between the $+C A D$ and $+P A D$ groups.

\begin{tabular}{lllllll}
\hline & $\begin{array}{l}\text { Overall } \\
(\mathrm{N}=75)\end{array}$ & $\begin{array}{l}\text { +CAD } \\
(\mathrm{N}=30)\end{array}$ & $\begin{array}{l}\text { +PAD } \\
(\mathrm{N}=5)\end{array}$ & $\begin{array}{l}\text { +CAD+PAD } \\
(\mathrm{N}=11)\end{array}$ & $\begin{array}{l}\text {-CAD-PAD } \\
(\mathrm{N}=29)\end{array}$ & P value \\
\hline Female & $32(45 \%)$ & $8(26.6 \%)$ & $3(60 \%)$ & $5(45 \%)$ & $16(55 \%)$ & 0.02 \\
\hline Age & $72.2 \pm 11.6$ & $75.3 \pm 7.4$ & $81.4 \pm 5.5$ & $75 \pm 8.8$ & $66.4 \pm 14.2$ & 0.08 \\
\hline BMI & $30.5 \pm 6.2$ & $30.3 \pm 5.1$ & $23.9 \pm 4.7$ & $29.5 \pm 4.7$ & $32.4 \pm 7.1$ & 0.01 \\
\hline Current Smoker & $10(13 \%)$ & $4(13 \%)$ & $1(20 \%)$ & $2(18 \%)$ & $3(10 \%)$ & 0.5 \\
\hline Former Smoker & $35(46 \%)$ & $17(57 \%)$ & 0 & $7(64 \%)$ & $11(38 \%)$ & 0.04 \\
\hline Hypertension & $65(87 \%)$ & $26(87 \%)$ & $4(80 \%)$ & $10(91 \%)$ & $24(86 \%)$ & 0.002 \\
\hline Hyperlipidemia & $50(67 \%)$ & $24(80 \%)$ & $3(60 \%)$ & $10(91 \%)$ & $12(41 \%)$ & 0.5 \\
\hline PVD & $16(21 \%)$ & 0 & $5(100 \%)$ & $11(100 \%)$ & 0 & \\
\hline CAD & $40(53 \%)$ & $30(100 \%)$ & NA & $11(100 \%)$ & 0 & \\
$-\quad$ STEMI & $10(13 \%)$ & $10(34 \%)$ & & $4(36 \%)$ & & \\
$-\quad$ NSTEMI & $9(12 \%)$ & $9(30 \%)$ & & $2(18 \%)$ & & 1 \\
\hline Diabetes & $32(43 \%)$ & $12(40 \%)$ & $2(40 \%)$ & $8(73 \%)$ & $9(31 \%)$ & 0.6 \\
$-\quad$ Oral & $19(25 \%)$ & $8(27 \%)$ & $2(40 \%)$ & $3(27 \%)$ & $6(21 \%)$ & 1 \\
$-\quad$ Insulin & $10(13 \%)$ & $4(13 \%)$ & 0 & $4(36 \%)$ & $2(7 \%)$ & 0.6 \\
\hline CKD & $20(27 \%)$ & $8(27 \%)$ & $1(20 \%)$ & $6(55 \%)$ & $5(17 \%)$ & 1 \\
\hline Neoplasm & $17(23 \%)$ & $8(27 \%)$ & 0 & $2(18 \%)$ & $9(31 \%)$ & 0.3 \\
\hline Aspirin & $50(67 \%)$ & $24(80 \%)$ & $2(40 \%)$ & $9(82 \%)$ & $15(52 \%)$ & 0.09 \\
\hline P2Y12i & $10(13 \%)$ & $7(23 \%)$ & $1(20 \%)$ & $2(18 \%)$ & $1(3 \%)$ & 1 \\
\hline Anticoagulation & $14(19 \%)$ & $4(13 \%)$ & $2(40 \%)$ & $3(27 \%)$ & $5(17 \%)$ & 0.1 \\
$-\quad$ NOAC & $8(11 \%)$ & $4(13 \%)$ & 0 & $1(9 \%)$ & $3(10 \%)$ & \\
$-\quad$ warfarin & $6(8 \%)$ & 0 & $2(40 \%)$ & $2(18 \%)$ & $2(7 \%)$ & $19(66 \%)$ \\
\hline Statin & $61(81 \%)$ & $27(90 \%)$ & $4(80 \%)$ & $100(100 \%)$ & 0.4 \\
\hline Beta-blockers & $63(84 \%)$ & $24(80 \%)$ & $4(80 \%)$ & $100(100 \%)$ & $24(83 \%)$ & 1 \\
\hline CCB & $26(35 \%)$ & $9(30 \%)$ & $2(40 \%)$ & $6(55 \%)$ & $9(31 \%)$ & 0.6 \\
\hline ACEI/ARB & $28(37 \%)$ & $10(33 \%)$ & $3(60 \%)$ & $2(18 \%)$ & $13(45 \%)$ & 0.3 \\
\hline
\end{tabular}

This distribution is similar to larger cohorts as reported in the PARTNERS registry with patients recruited from primary care offices [15] or in randomized trials [9]. The higher percentage of CAD patients in our study reflects the cardiology practice setting. This cohort reflects a typical cardiology outpatient population with a very high prevalence of hypertension (>80\% with each group). Not surprisingly the $+C A D$ cohort was predominantly male $(n=22,73 \%)$ in comparison to the other groups $(p=0.02)$. Also, not surprisingly, patients with $+C A D+P A D$ were more likely to 
have DM and CKD (73\% and 55\% respectively). This cohort thus represents a typical outpatient cardiovascular disease cohort with chronic stable disease (no recent acute events).

\section{Biomarkers for platelet activation is highest in CAD patients (Figure 2)}

Soluble P-selectin (sP-sel) level was higher in all patients compared to healthy young individuals $(402 \pm 38 \mathrm{pg} / \mathrm{mg}$ protein), with the highest levels in the +CAD (861 $61 \mathrm{pg} / \mathrm{mg}$ protein), +PAD (870 $\pm 152 \mathrm{pg} / \mathrm{mg}$ protein), and +CAD+PAD (816 $\pm 82 \mathrm{pg} / \mathrm{mg}$ protein) groups. Soluble CD40 ligand (sCD40L) level was significantly higher only in patients with documented CAD; +CAD (1126 \pm 111 $\mathrm{pg} / \mathrm{mg}$ protein) and +CAD+PAD (1125 $\pm 219 \mathrm{pg} / \mathrm{mg}$ protein), compared to +PAD (616 $206 \mathrm{pg} / \mathrm{mg}$ protein) and -CAD-PAD (768 $\pm 11 \mathrm{pg} / \mathrm{mg}$ protein 5$)$. Taken together this suggests CAD patients may have the most active platelets.

Figure 2. Presence of Coronary Artery Disease (CAD) causes increase in the level of platelet activation markers as compared to the young healthy controls. Platelet activation markers were measured using ELISA in the plasma from the patients (A) Soluble P-selectin level, (B) Soluble CD40L levels. The results are presented as mean \pm SEM. $p$ - values as calculated by One-Way ANOVA ${ }^{*} p<0.05,{ }^{* *} p<0.005,{ }^{* * *} p<0.001$.

A

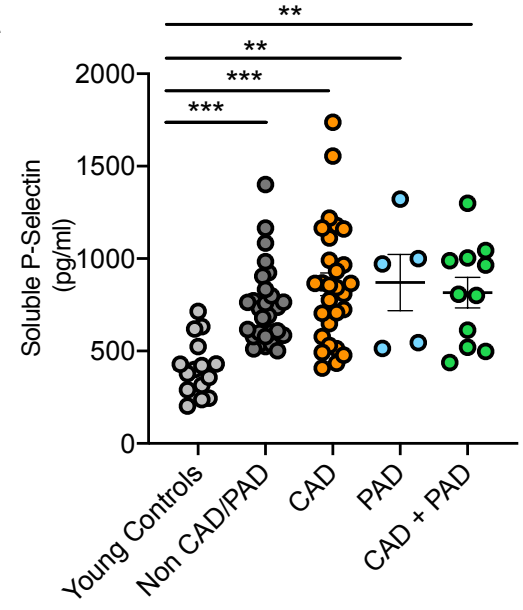

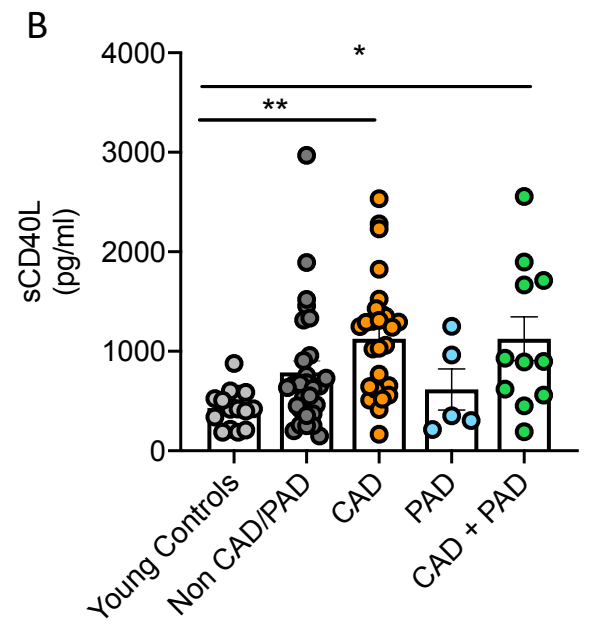




\section{Biomarkers of oxidative stress is highest in CAD patients (Figure 3)}

Platelet oxidative stress is widely accepted to be closely related to platelet activation, with higher ROS being associated with increased platelet activation [16]. As ROS is transient, measurements can be problematic and thus other indices reflecting end results of oxidation such as lipid peroxidation and protein carbonylation are needed. As expected, ROS levels are increased in elderly patients regardless of disease status. Although not statistically different, it is noteworthy that again, the highest levels were in the CAD groups (+CAD and +CAD+PAD) with no differences between the +PAD and -CAD-PAD cohorts. This same trend was apparent for both lipid peroxidation and protein carbonylation (Figure 3).

Figure 3. Platelets from the CAD cohort show differences in the redox homeostasis. Freshly isolated platelets from citrated blood were used for all experiments. A. Intracellular ROS in platelets measured by the fluorescence emission of the dye DCFH-DA. B. Intracellular lipid peroxidation in platelets measured using the TBARS assay. C. Protein carbonylation levels measured based on the derivatization with 2,4, DNPH assayed. D-E. Catalase and SOD activity was measured and normalized to the protein concentration of each platelet sample. Values expressed as Mean \pm SEM. ${ }^{*} p<0.05 ;{ }^{*} p<0.005 ;{ }^{* *} p<0.005$ determined by one-way ANOVA with Bonferroni post hoc analysis.

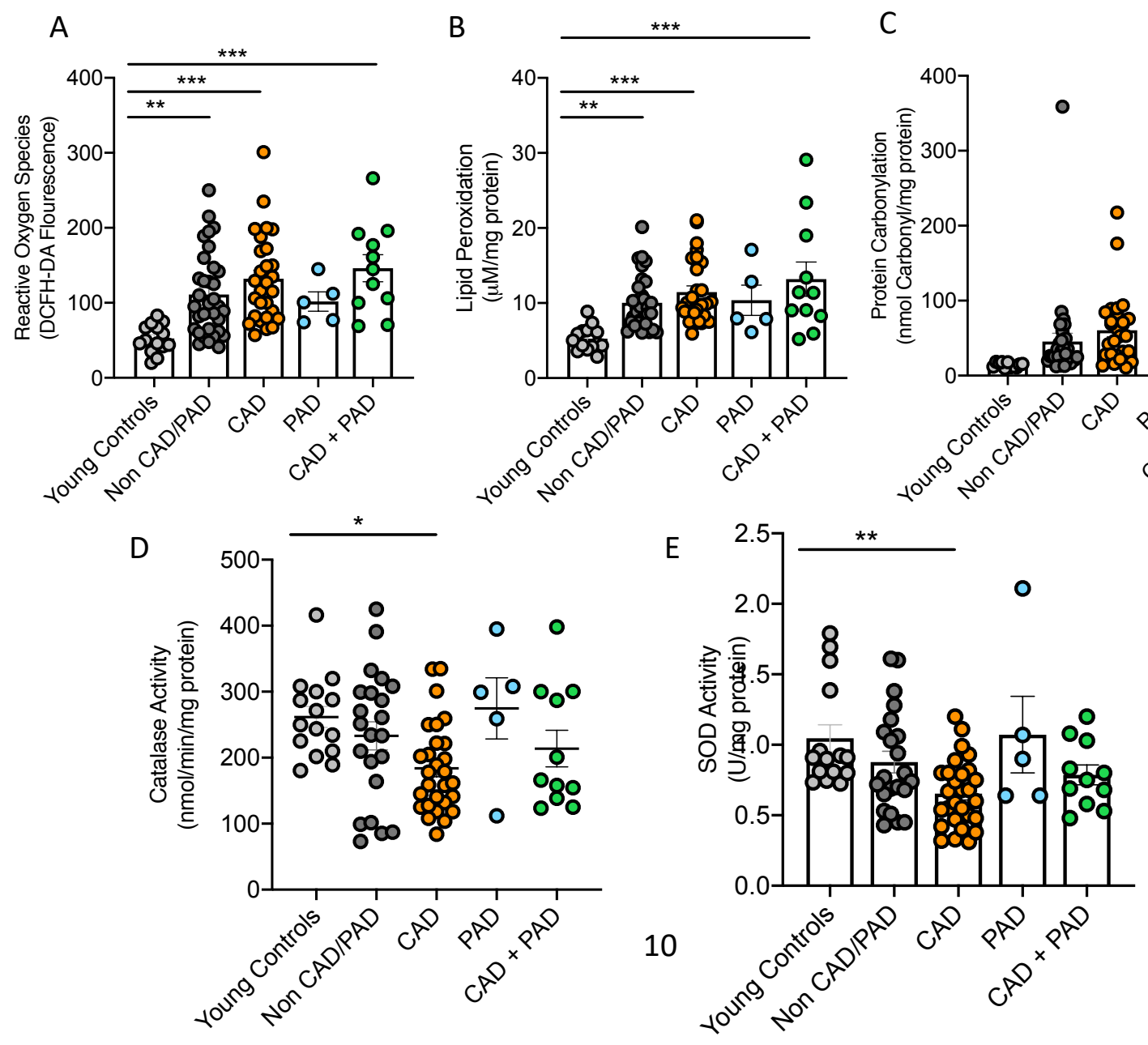


These results closely reflect and support the platelet activation data. The interesting question then arises as to the antioxidant status of the platelets. Particularly as this may explain in part the etiology of the oxidative changes. Platelet CAT and SOD levels were lowest in patients with CAD (+CAD and +CAD+PAD). This again is consistent with and supports the high ROS and high platelet activation observed with the +CAD and +CAD+PAD groups.

In order to provide statistical support, we performed correlation tests and thus are able to compare results from individual patients rather than cohort means. For +CAD there was a statistically significant negative correlation between SCD40L (activation) - SOD level (antioxidant) ( $r=-0.4, p=0.01$ ) and positive correlation of sP-Selectin (activation) and lipid peroxidation (oxidant) $(r=0.5, p=0.04)$ (Table 2). Moreover, for $+P A D$ there was a negative correlation between the SCD40L (activation) - catalase (antioxidant) levels ( $r=-0.89, p=0.016)$ and positive correlation of SCD40L (activation) - lipid peroxidation (oxidant) $(r=0.9, p=0.02)$. Taken together this supports CAD as being associated with low platelet antioxidants, high oxidative stress and high platelet activation. In contrast, PAD may be the opposite with high antioxidants, low oxidative stress and low platelet activation.

Table 2. Correlation coefficients ( $r$ ) and p-values ( $p$ ) of correlations between sP-selectin, sCD40L, catalase and SOD activity in CAD and PAD.

\begin{tabular}{|c|c|c|}
\hline Parameter & $r$ & $\mathbf{p}$ \\
\hline \multicolumn{3}{|c|}{ Coronary artery disease } \\
\hline sP-selectin-sCD40L & 0.49 & 0.004 \\
\hline $\begin{array}{l}\text { sP-selectin-lipid } \\
\text { peroxidation }\end{array}$ & 0.48 & 0.02 \\
\hline sCD40L-SOD & -0.44 & 0.01 \\
\hline Catalase-SOD & 0.61 & 0.0003 \\
\hline $\begin{array}{l}\text { Catalase-lipid } \\
\text { peroxidation }\end{array}$ & -0.44 & 0.02 \\
\hline $\begin{array}{l}\text { Catalase-protein } \\
\text { carbonylation }\end{array}$ & -0.47 & 0.02 \\
\hline \multicolumn{3}{|c|}{ Peripheral artery disease } \\
\hline sCD40L-catalase & -0.89 & 0.01 \\
\hline $\begin{array}{l}\text { sCD40L-lipid } \\
\text { peroxidation }\end{array}$ & 0.90 & 0.02 \\
\hline
\end{tabular}


Inflammatory biomarkers assessment (Figure 4)

To further support potential differences in stressors between the groups we assessed for some basic inflammatory biomarkers (Figure 4).

Figure 4. Presence of CAD and/or PAD mediates an increase in the inflammatory cytokines. A. Interleukin-1 levels. B. Interleukin- 6 levels. C. Tumor Necrosis Factor- $\alpha$ levels. Values expressed as Mean \pm SEM. Statistical significance ${ }^{*} p<0.05 ;{ }^{* *} p<0.005 ;{ }^{* * *} p<0.005$ determined by one-way ANOVA with Bonferroni post hoc analysis.

A

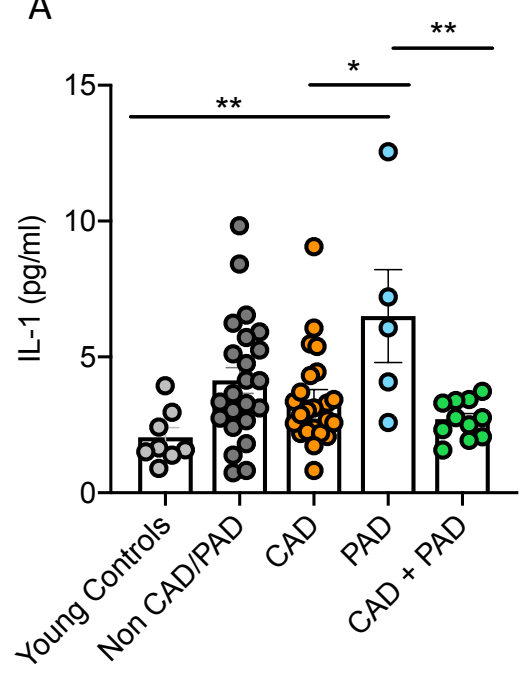

B
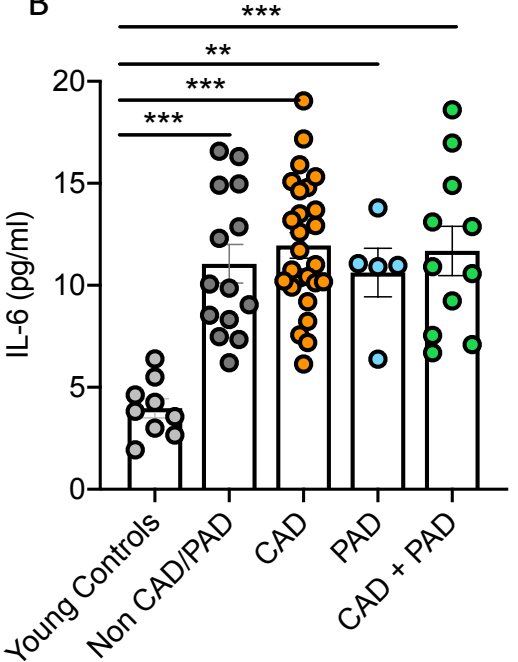

C

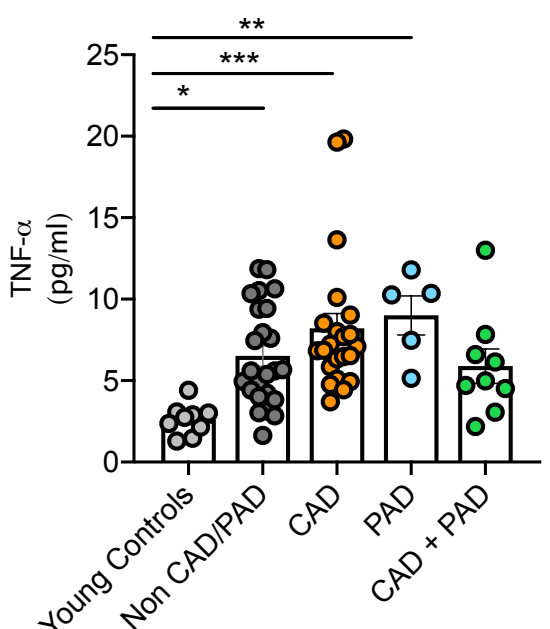

Again, and not surprisingly they were increased in all older cohorts compared to the young group but again, what was significantly different from the younger cohort and also between groups was key. Most notable, interleukin-1 $\beta$ (IL1 $\beta$ ) levels were higher in +PAD compared to +CAD and even +CAD+PAD patients, while interleukin-6 (IL6) and TNF- $\alpha$ levels were higher in all patients compared to the healthy young group. Taken together, although small in numbers, the combined results support a differential and consistent stressor profile in patients with CAD versus PAD where platelet activation may play a more important role in CAD with increased platelet oxidative stress. PAD in contrast may be more of a more IL1 $\beta$ mediated inflammatory process, with less activated platelet involvement (Figure 5). 
Figure 5. Summary of findings. The two disease processes CAD and PAD are represented diagrammatically. Both platelets and inflammation contribute to the disease processes however there may be differential contribution with platelet activation/ROS playing more of a role in CAD and IL1b-mediated pathology and possible hypercoagulable state playing a prominent role in PAD pathology. This may explain differences in outcomes and response to therapies for CAD and PAD.

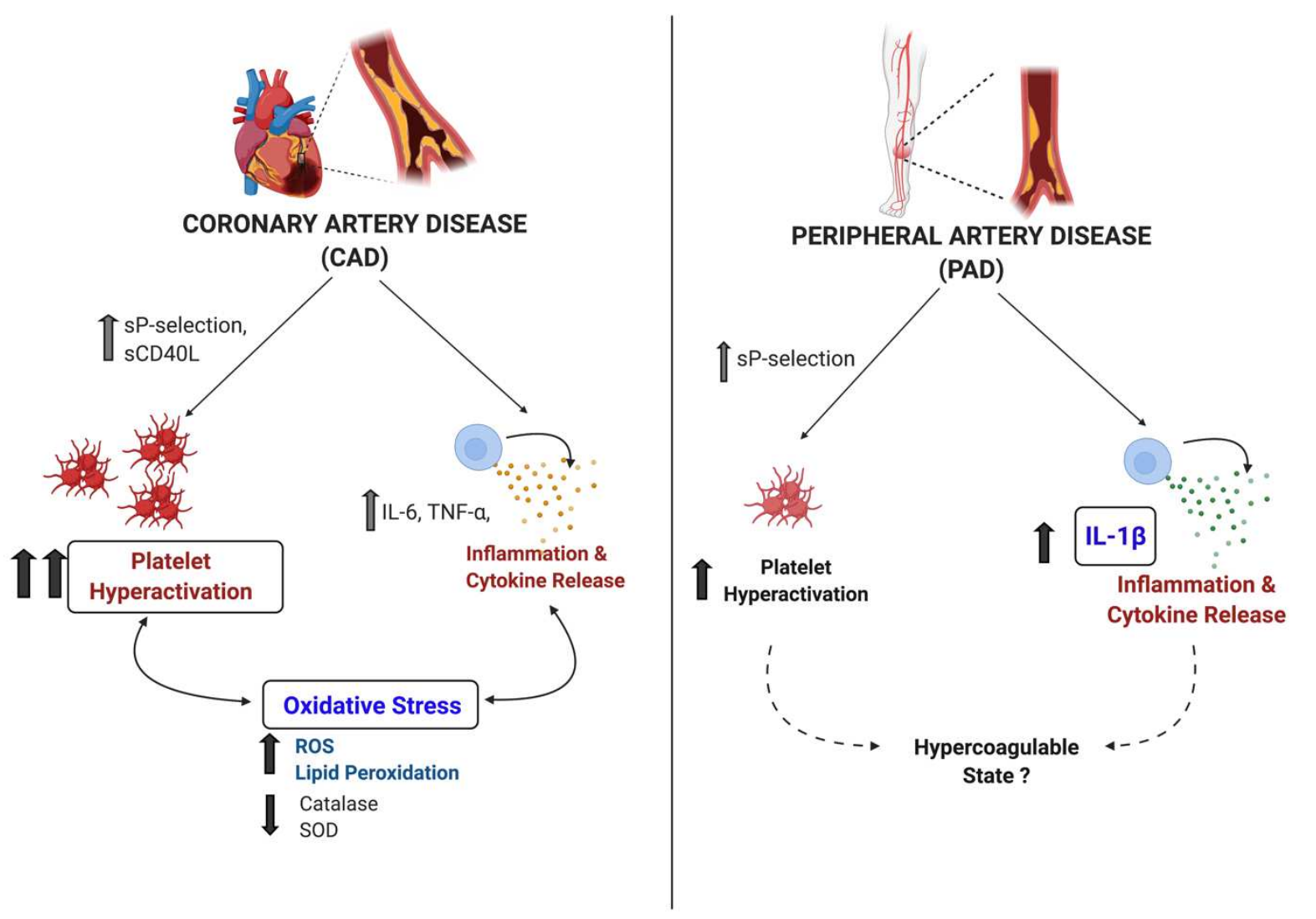

\section{DISCUSSION}

Chronic CAD and PAD are both considered atheromatous diseases, however, multiple differences besides disease location, have been observed including mortality and morbidity, and response to medical and interventional therapies. Part of the difference between the two diseases may be related to the platelet phenotype, a recognized major contributor to the atherothrombosis process $[5,6]$. To explore if platelets are phenotypically different between CAD and PAD, we extensively investigated platelet phenotype and key plasma inflammatory markers in stable chronic $C A D$ and $P A D$, in an outpatient setting. Based upon prior experience with platelet analysis 
two important approaches were adopted for this study. Firstly, we excluded acute vascular events such as myocardial infarction or critical limb ischemia as they could contaminate oxidation-inflammatory assessments with transient elevations in platelet and inflammatory markers. The focus was thus on the development of chronic disease. Secondly, because of extensive interpatient variability and the sometimes transient nature of oxidation-inflammatory markers, it was imperative to perform multiple assays for related biomarkers to compare the diseased and non-diseased cohorts to a young cohort, highlighting statistical differences and focusing on consistent and greatest changes from the mean.

Platelet activation is associated with aging, particularly in CAD patients. Elevations in sP-sel and SCD40L within the -CAD-PAD group could be attributed to other underlying conditions (hypertension, hyperlipidemia, diabetes, smoking) known to be associated with increased in sPsel [17-20]. However, we found higher SCD40L level only in the presence of CAD. Interestingly, we did not find an elevation in SCD40L in +PAD. Prior studies of SCD40L in PAD, found elevated levels in patients with acute disease including intermittent claudication or critical limb ischemia [21]. Our patients were not acutely symptomatic. Clinical evidence supports the use of aspirin only in symptomatic PAD, suggesting possible active platelets during an acute process [22]. Also, SCD40L is known to decrease with medical therapy [23] and all our patients received optimal therapies PAD. In addition, in patients with chronic inflammatory conditions, like congestive heart failure [24] or hypercholesterolemia [25], normal levels of SCD40L have been documented. In these conditions, there is upregulation of the membrane bound CD40 and a pseudonormalization of the SCD40L levels, in contrast with the acute conditions, where platelet stimulation, with ADP, thrombin or collagen, CD40L is translocated to the membrane and cleaved in a time-dependent manner, with increase in SCD40L levels during the acute phase. Thus, the focus was on chronic disease as distinct from acute on chronic disease.

Reactive oxygen species (ROS) are produced by living cells as normal cellular metabolic byproduct. Under conditions of stress, there is an excessive production of ROS which can trigger cellular defense mechanisms including autophagy [26] and inflammatory responses and excessive ROS 
levels can lead to apoptosis [27]. Antioxidant systems are present in the cells to neutralize the ROS levels and maintain the redox homeostasis. This consists of enzymatic antioxidants such as SOD, CAT and glutathione peroxidases (GPxs), thioredoxin (Trx) as well as the non-enzymatic antioxidants which collectively reduce oxidative state. ROS plays a central role in atherosclerosis [28]. Due to higher incidence of traditional and novel cardiovascular risk factors, PAD has a higher morbidity and mortality compared to $\operatorname{CAD}[29,30]$ and thus ROS was expected to be higher. Unexpectedly, we found higher platelet ROS levels in CAD compared to PAD. This may be explained in part by a lower ability to remove the ROS due to reduced CAT and SOD enzymatic activity. The reason for the reduced antioxidant enzymes requires further exploration.

To our knowledge this the first study directly comparing platelet ROS in CAD versus PAD. A prior study of ROS in platelets from patients with CAD reported higher levels compared to controls [31]. In addition, another study comparing plasma ROS levels did not find a significant difference between patients with PAD and healthy controls [32]. These results are in line with the current understanding of atherosclerosis in which higher levels of ROS in endothelial cells contribute to the formation and progression of atherosclerosis [33]. We show here that similar changes are present in the platelets from patients with CAD. However, in PAD patients, platelets may have a different phenotype with no increase in ROS or lipid peroxidation, and no significant differences in CAT and SOD activity as compared to the -CAD-PAD group. Our findings add to the growing body of evidence that the pathophysiology of PAD may be different compared with CAD, suggesting a lesser role for ROS and platelets in PAD. This claim is supported by recent autopsy findings of limbs with PAD and critical limb ischemia showing that the lesion composition in PAD was very different from $C A D$, with medial calcification and intimal thickening, but absence of lipids [34, 35]. Also, platelets react differently to collagen and thrombin, with only collagen increasing the $\mathrm{H}_{2} \mathrm{O}_{2}$ production [36]. PAD is known to increase coagulation activity and thrombin $[37,38]$, while atherosclerotic coronary disease appear collagen driven [39].

Atherosclerosis is an inflammatory disease. Pathophysiologic, genetic and basic and clinical evidence implicate vascular inflammation in the initiation and progression of atherosclerosis. IL- 
1, IL-6, TNF- $\alpha$ have been studied extensively over the last several decades. In our cohort the levels of IL-1 differ significantly, being highest in the +PAD group. IL-1 $\beta$ decreases the level of thrombomodulin resulting in impaired activation of protein $\mathrm{C}$, with subsequent hypercoagulable state [40]. In addition, down-regulation of thrombomodulin decreases the thrombin-mediated formation of activated protein $\mathrm{C}$ and mitigates its anti-inflammatory effects [41]. In a recent histopathologic study, atheromas were found in only $23 \%$ of 176 upper and lower leg arteries from 60 patients with PAD and amputated limbs [34]. A possible explanation, at least for below knee disease, was offered by another histopathologic examination of 12 legs from 8 cadavers yielding 2,987 sections [35], in which at least half of the occluded arteries below the knee were embolic in origin, suggesting a hypercoagulable state. The higher level of IL-1 $\beta$ in PAD, with a consequent hypercoagulable state, coupled with lower levels of ROS and lipid peroxidation, offers a possible molecular explanation of these histopathologic findings. PAD may be more of a more IL1ß-mediated inflammatory disease process with reduced platelet involvement in comparison to CAD. The hypercoagulable state in PAD is also supported by recent data from large clinical trials [9], in which low dose anticoagulation in addition to antiplatelet therapy significantly decreased the risk of lower extremity thrombosis and stroke but not myocardial infarction. In addition, the improved outcome in PAD with P2Y12 vs aspirin [8] could also be related to enhanced inhibition of thrombin generation by P2Y12 as opposed to aspirin [42] with subsequent improved coagulable state.

In conclusion, our data suggest very different platelet oxidation-inflammatory phenotypes in CAD versus PAD. In our cohort, platelets from CAD patients had higher ROS and lower antioxidant enzyme activity compared to PAD. Further studies to expand these findings are needed. However, it underlines the importance of clinical phenotype when studying platelets. Given the large overlap between CAD and PAD [43], future research should carefully document the clinical substrate when evaluating platelets function. 


\section{Limitations}

The patients were recruited from a single cardiology clinic, which could introduce bias. The majority of patients classified as not having disease were investigated with stress test and/or coronary angiography (75\%), tests with high specificity for significant coronary artery disease. However, these tests do not exclude non-significant CAD, especially the stress testing. Moreover, not all patients were investigated for PAD, and it is known that a large number of patients can have asymptomatic PAD. A rigorous study to exclude patients with angiography and an increase number is needed. We have however provided detailed platelet oxidation status evidence suggesting important pathophysiological differences.

\section{REFERENCES}

1. Arias E, X.J., United States life tables, 2017. National Vital Statistics Reports. 2019, Hyattsville, MD: National Center for Health Statistics.

2. Hansson, G.K., et al., Innate and adaptive immunity in the pathogenesis of atherosclerosis. Circ Res, 2002. 91(4): p. 281-91.

3. Libby, P., P.M. Ridker, and G.K. Hansson, Progress and challenges in translating the biology of atherosclerosis. Nature, 2011. 473(7347): p. 317-25.

4. Chatterjee, M. and M. Gawaz, Platelets in Atherosclerosis, in Platelets in Thrombotic and Non-Thrombotic Disorders: Pathophysiology, Pharmacology and Therapeutics: an Update, P. Gresele, et al., Editors. 2017, Springer International Publishing: Cham. p. 9931013.

5. Huo, Y., et al., Circulating activated platelets exacerbate atherosclerosis in mice deficient in apolipoprotein E. Nat Med, 2003. 9(1): p. 61-7.

6. Lievens, D., et al., Platelet CD4OL mediates thrombotic and inflammatory processes in atherosclerosis. Blood, 2010. 116(20): p. 4317-27.

7. Steg, P.G., et al., One-year cardiovascular event rates in outpatients with atherothrombosis. Jama, 2007. 297(11): p. 1197-206.

8. A randomised, blinded, trial of clopidogrel versus aspirin in patients at risk of ischaemic events (CAPRIE). CAPRIE Steering Committee. Lancet, 1996. 348(9038): p. 1329-39.

9. Gurbel, P.A., et al., Combination Antiplatelet and Oral Anticoagulant Therapy in Patients With Coronary and Peripheral Artery Disease. Circulation, 2019. 139(18): p. 2170-2185.

10. Nambi, V., et al., Differences in responses of platelets to fluid shear stress in patients with peripheral artery disease (PAD) and coronary artery disease (CAD). Platelets, 2009. 20(3): p. 199-205.

11. Keating, F.K., et al., Relation of augmented platelet reactivity to the magnitude of distribution of atherosclerosis. Am J Cardiol, 2004. 94(6): p. 725-8. 
12. Gremmel, T., et al., Response to antiplatelet therapy and platelet reactivity to thrombin receptor activating peptide- 6 in cardiovascular interventions: Differences between peripheral and coronary angioplasty. Atherosclerosis, 2014. 232(1): p. 119-24.

13. Schmidt, R.A., et al., The platelet phenotype in patients with ST-segment elevation myocardial infarction is different from non-ST-segment elevation myocardial infarction. Transl Res, 2018. 195: p. 1-12.

14. Jain, K., et al., Age associated non-linear regulation of redox homeostasis in the anucleate platelet: Implications for CVD risk patients. EBioMedicine, 2019. 44: p. 28-40.

15. Hirsch, A.T., et al., Peripheral arterial disease detection, awareness, and treatment in primary care. Jama, 2001. 286(11): p. 1317-24.

16. Krötz, F., et al., $N A D(P) H$ oxidase-dependent platelet superoxide anion release increases platelet recruitment. Blood, 2002. 100(3): p. 917-24.

17. Harrison, D., et al., Role of oxidative stress in atherosclerosis. Am J Cardiol, 2003. 91(3a): p. 7a-11a.

18. Togliatto, G., G. Lombardo, and M.F. Brizzi, The Future Challenge of Reactive Oxygen Species (ROS) in Hypertension: From Bench to Bed Side. Int J Mol Sci, 2017. 18(9).

19. Qiao, J., et al., Regulation of platelet activation and thrombus formation by reactive oxygen species. Redox Biol, 2018. 14: p. 126-130.

20. Blann, A.D., S.K. Nadar, and G.Y. Lip, The adhesion molecule P-selectin and cardiovascular disease. Eur Heart J, 2003. 24(24): p. 2166-79.

21. Blann, A.D., et al., Soluble CD4OL in peripheral artery disease. Relationship with disease severity, platelet markers and the effects of angioplasty. Thromb Haemost, 2005. 93(3): p. 578-83.

22. Gerhard-Herman, M.D., et al., 2016 AHA/ACC Guideline on the Management of Patients With Lower Extremity Peripheral Artery Disease. A Report of the American College of Cardiology/American Heart Association Task Force on Clinical Practice Guidelines, 2017. 69(11): p. e71-e126.

23. Choudhury, A., et al., Soluble CD40 ligand, platelet surface CD40 ligand, and total platelet CD40 ligand in atrial fibrillation: relationship to soluble P-selectin, stroke risk factors, and risk factor intervention. Chest, 2008. 134(3): p. 574-581.

24. Stumpf, C., et al., Enhanced levels of CD154 (CD40 ligand) on platelets in patients with chronic heart failure. European Journal of Heart Failure, 2003. 5(5): p. 629-637.

25. Garlichs, C.D., et al., Upregulation of CD40 and CD40 ligand (CD154) in patients with moderate hypercholesterolemia. Circulation, 2001. 104(20): p. 2395-400.

26. Lee, S.H., et al., Inducing mitophagy in diabetic platelets protects against severe oxidative stress. EMBO Mol Med, 2016. 8(7): p. 779-95.

27. Leytin, V., Apoptosis in the anucleate platelet. Blood Rev, 2012. 26(2): p. 51-63.

28. Förstermann, U., N. Xia, and H. Li, Roles of Vascular Oxidative Stress and Nitric Oxide in the Pathogenesis of Atherosclerosis. Circ Res, 2017. 120(4): p. 713-735.

29. Nguyen, C.H., et al., Entering Cardiac Rehabilitation With Peripheral Artery Disease: A RETROSPECTIVE COMPARISON TO CORONARY ARTERY DISEASE. J Cardiopulm Rehabil Prev, 2020. 40(4): p. 255-262. 
30. Banerjee, A., F.G. Fowkes, and P.M. Rothwell, Associations between peripheral artery disease and ischemic stroke: implications for primary and secondary prevention. Stroke, 2010. 41(9): p. 2102-7.

31. Buczyński, A., et al., Changes in antioxidant enzymes activities, aggregability and malonyldialdehyde concentration in blood platelets from patients with coronary heart disease. Atherosclerosis, 1993. 100(2): p. 223-8.

32. Jandrić-Balen, M., et al., Antioxidant enzymes activity in patients with peripheral vascular disease, with and without presence of diabetes mellitus. Coll Antropol, 2003. 27(2): p. 735-43.

33. Nowak, W.N., et al., Reactive Oxygen Species Generation and Atherosclerosis. Arterioscler Thromb Vasc Biol, 2017. 37(5): p. e41-e52.

34. O'Neill, W.C., et al., Prevalence of nonatheromatous lesions in peripheral arterial disease. Arterioscler Thromb Vasc Biol, 2015. 35(2): p. 439-47.

35. Torii, S., et al., Histopathologic Characterization of Peripheral Arteries in Subjects With Abundant Risk Factors: Correlating Imaging With Pathology. JACC Cardiovasc Imaging, 2019. 12(8 Pt 1): p. 1501-1513.

36. Del Principe, D., et al., Hydrogen peroxide is an intermediate in the platelet activation cascade triggered by collagen, but not by thrombin. Thromb Res, 1991. 62(5): p. 365-75.

37. Olie, R.H., P.E.J. van der Meijden, and H. Ten Cate, The coagulation system in atherothrombosis: Implications for new therapeutic strategies. Res Pract Thromb Haemost, 2018. 2(2): p. 188-198.

38. Kleinegris, M.F., et al., Increased Clot Formation in the Absence of Increased Thrombin Generation in Patients with Peripheral Arterial Disease: A Case-Control Study. Front Cardiovasc Med, 2017. 4: p. 23.

39. Davies, M.J., The pathophysiology of acute coronary syndromes. Heart (British Cardiac Society), 2000. 83(3): p. 361-366.

40. Bester, J. and E. Pretorius, Effects of IL-16, IL-6 and IL-8 on erythrocytes, platelets and clot viscoelasticity. Scientific Reports, 2016. 6(1): p. 32188.

41. Riewald, M., et al., Activation of endothelial cell protease activated receptor 1 by the protein C pathway. Science, 2002. 296(5574): p. 1880-2.

42. Perzborn, E., S. Heitmeier, and V. Laux, Effects of Rivaroxaban on Platelet Activation and Platelet-Coagulation Pathway Interaction: In Vitro and In Vivo Studies. J Cardiovasc Pharmacol Ther, 2015. 20(6): p. 554-62.

43. Criqui, M.H. and V. Aboyans, Epidemiology of peripheral artery disease. Circ Res, 2015. 116(9): p. 1509-26. 


\section{Authors Contributions}

$\mathrm{CNI}$ recruited the patients. $\mathrm{KJ}$ and TT performed the experiments with oversight and assistance of JH. KJ, TT, JH designed the experiments. CNI coordinated the project. KJ, TT, JH, CNI contributed to the writing of the manuscript.

\section{Competing interests}

None.

\section{Funding}

Study funded by NIH-NHLBI, RO1-HL122815, RO1-HL115247 and R01-HL150515 to JH. 


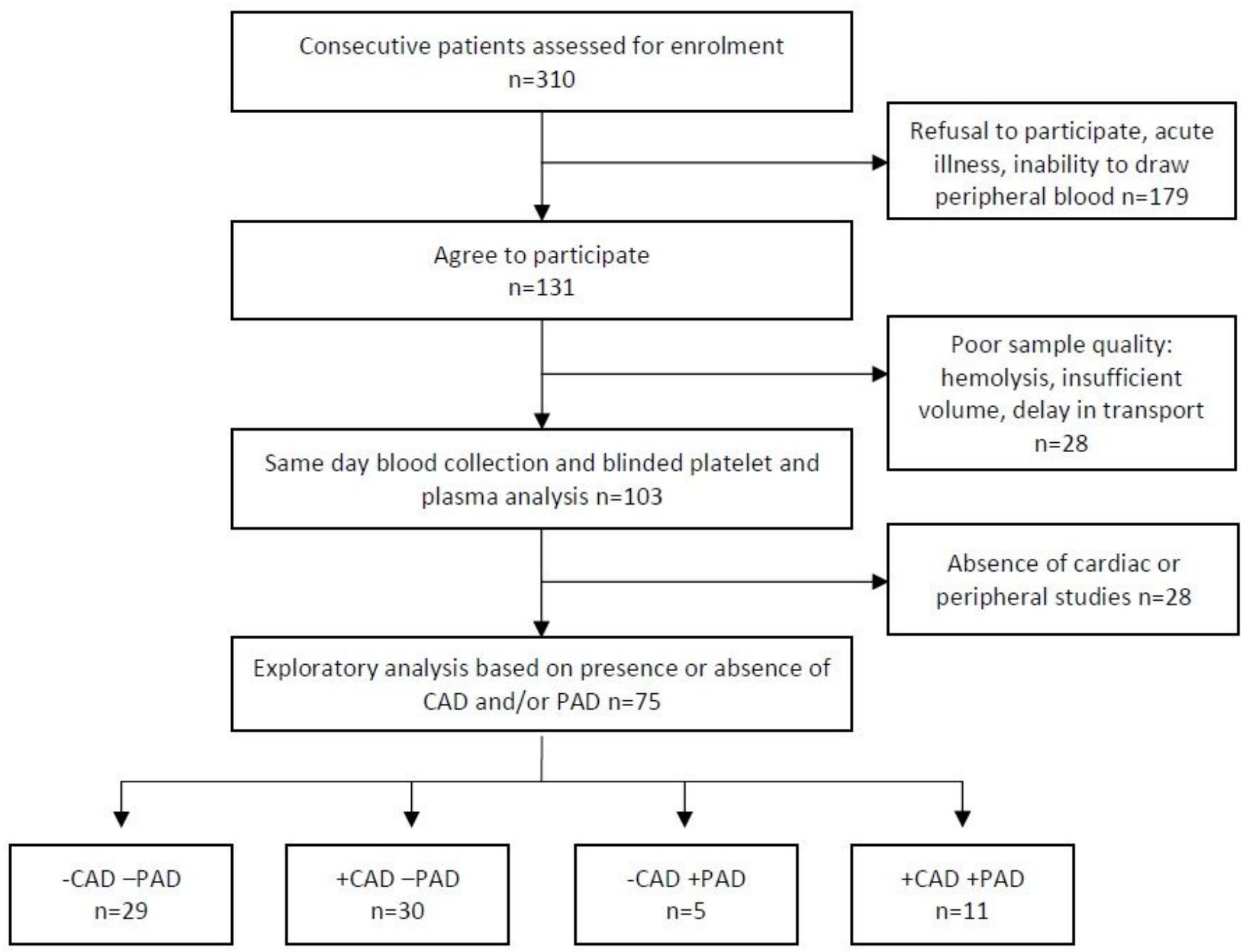

\section{Figure 1}

Schematic Overview of the study. Flow diagram outlining the patient screening and enrollment. 



Figure 2

Presence of Coronary Artery Disease (CAD) causes increase in the level of platelet activation markers as compared to the young healthy controls. Platelet activation markers were measured using ELISA in the plasma from the patients (A) Soluble P-selectin level, (B) Soluble CD 40L levels. The results are presented as mean \pm SEM. $p$ - values as calculated by One-Way ANOVA ${ }^{*} p<0.05,{ }^{* *} p<0.005,{ }^{* *} p<0.001$. 


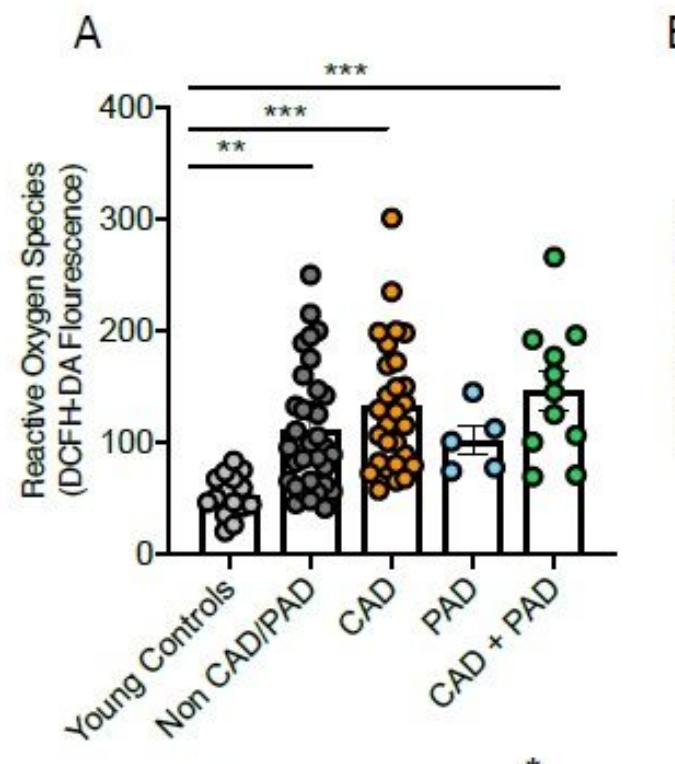

B

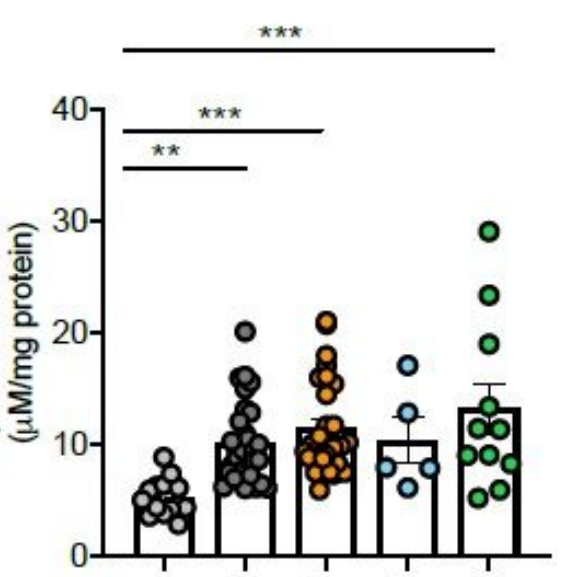

C
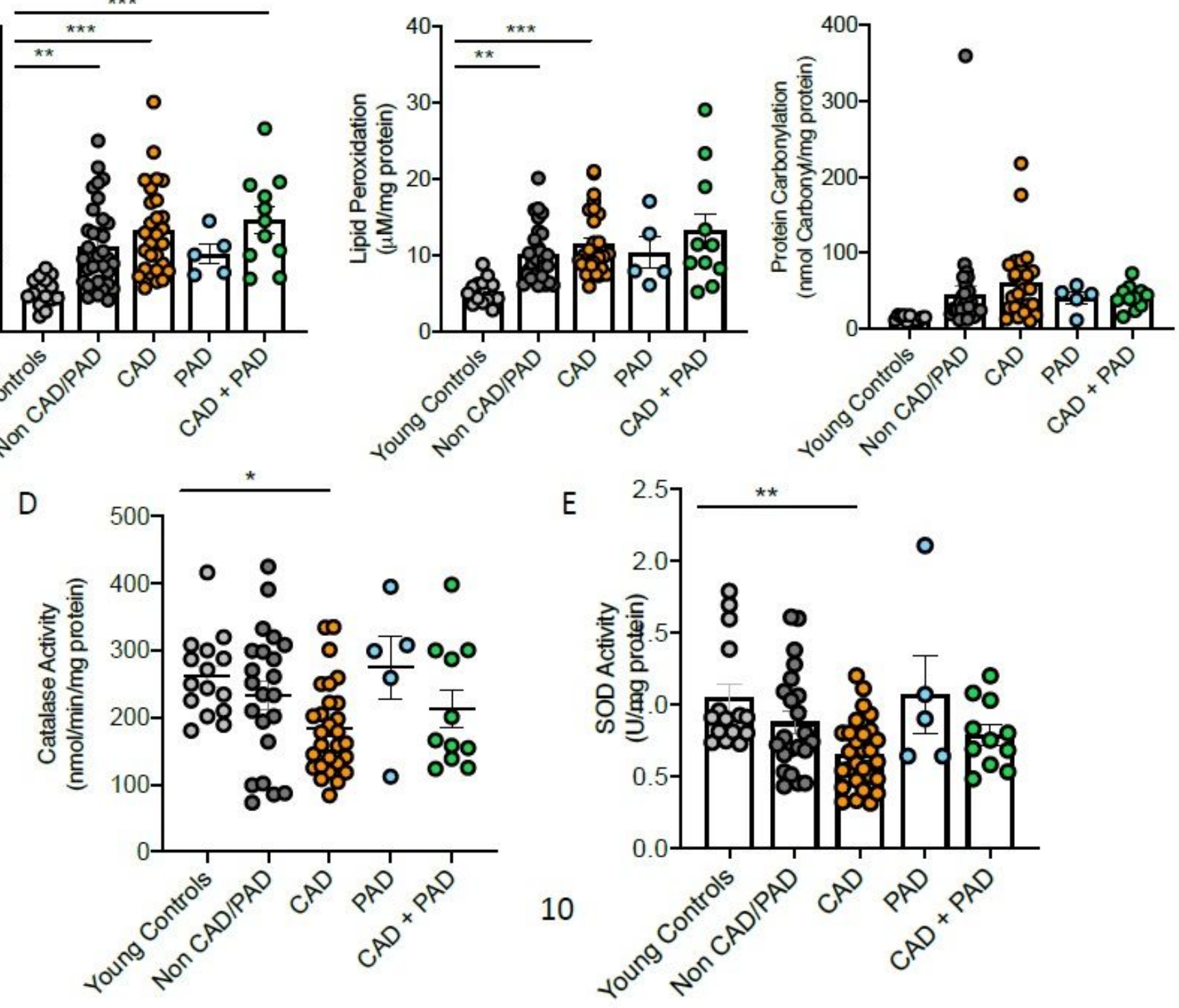

10

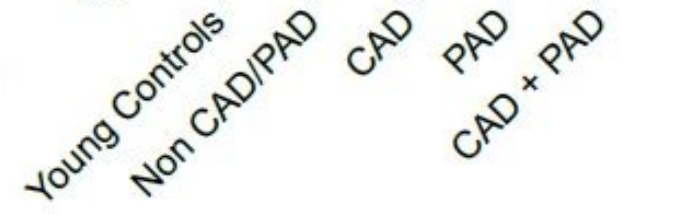

Figure 3

Platelets from the CAD cohort show differences in the redox homeostasis. Freshly isolated platelets from citrated blood were used for all experiments. A. Intracellular ROS in platelets measured by the fluorescence emission of the dye DCFH-DA. B. Intracellular lipid peroxidation in platelets measured using the TBARS assay. C. Protein carbonylation levels measured based on the derivatization with 2,4, DNPH assayed. D-E. Catalase and SOD activity was measured and normalized to the protein concentration of each platelet sample. Values expressed as Mean \pm SEM. ${ }^{*} p<0.05$; ${ }^{* \star} p<0.005$; ${ }^{* \star} p<0.005$ determined by one-way ANOVA with Bonferroni post hoc analysis. 

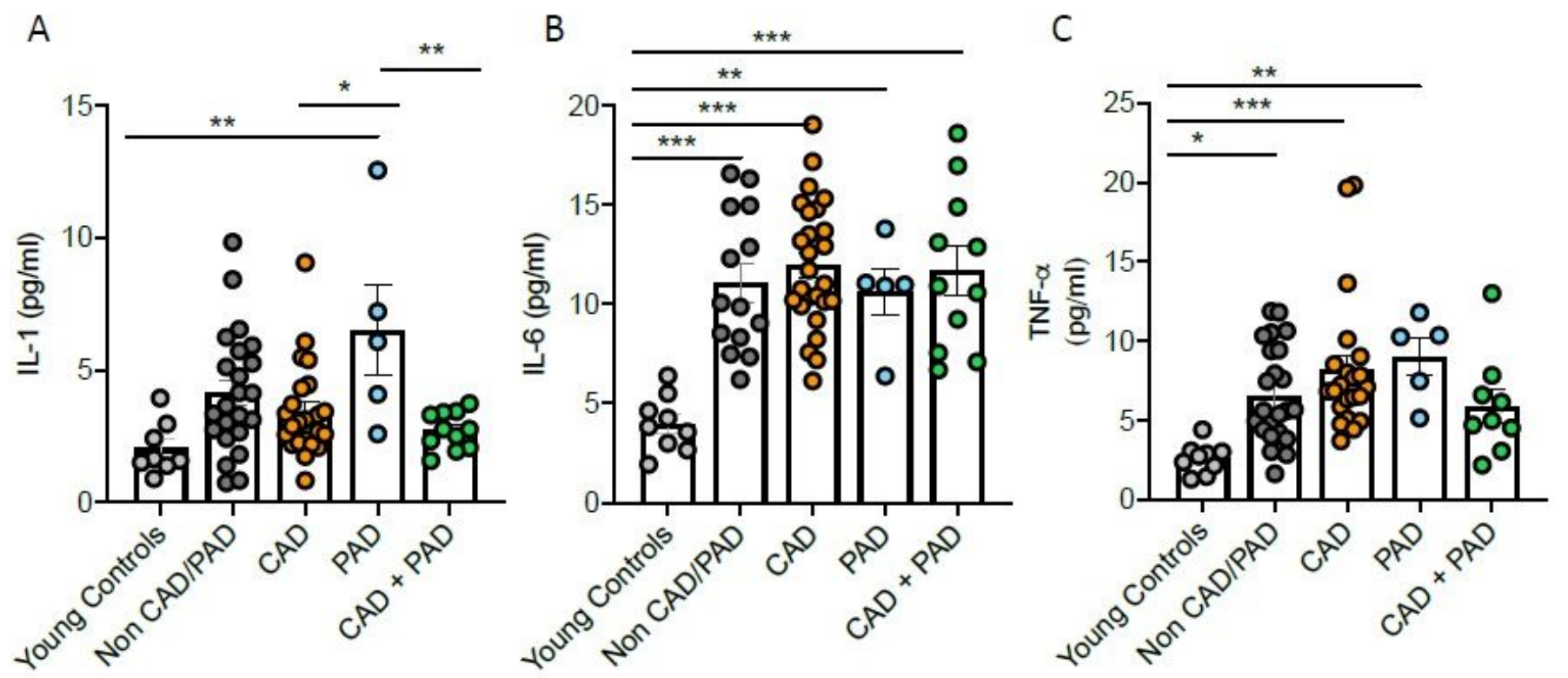

Figure 4

Presence of CAD and/or PAD mediates an increase in the inflammatory cytokines. A. Interleukin-1 levels. B. Interleukin-6 levels. C. Tumor Necrosis Factor-a levels. Values expressed as Mean \pm SEM. Statistical significance ${ }^{*} p<0.05 ;{ }^{* \star} p<0.005 ; * \star * p<0.005$ determined by one-way ANOVA with Bonferroni post hoc analysis. 


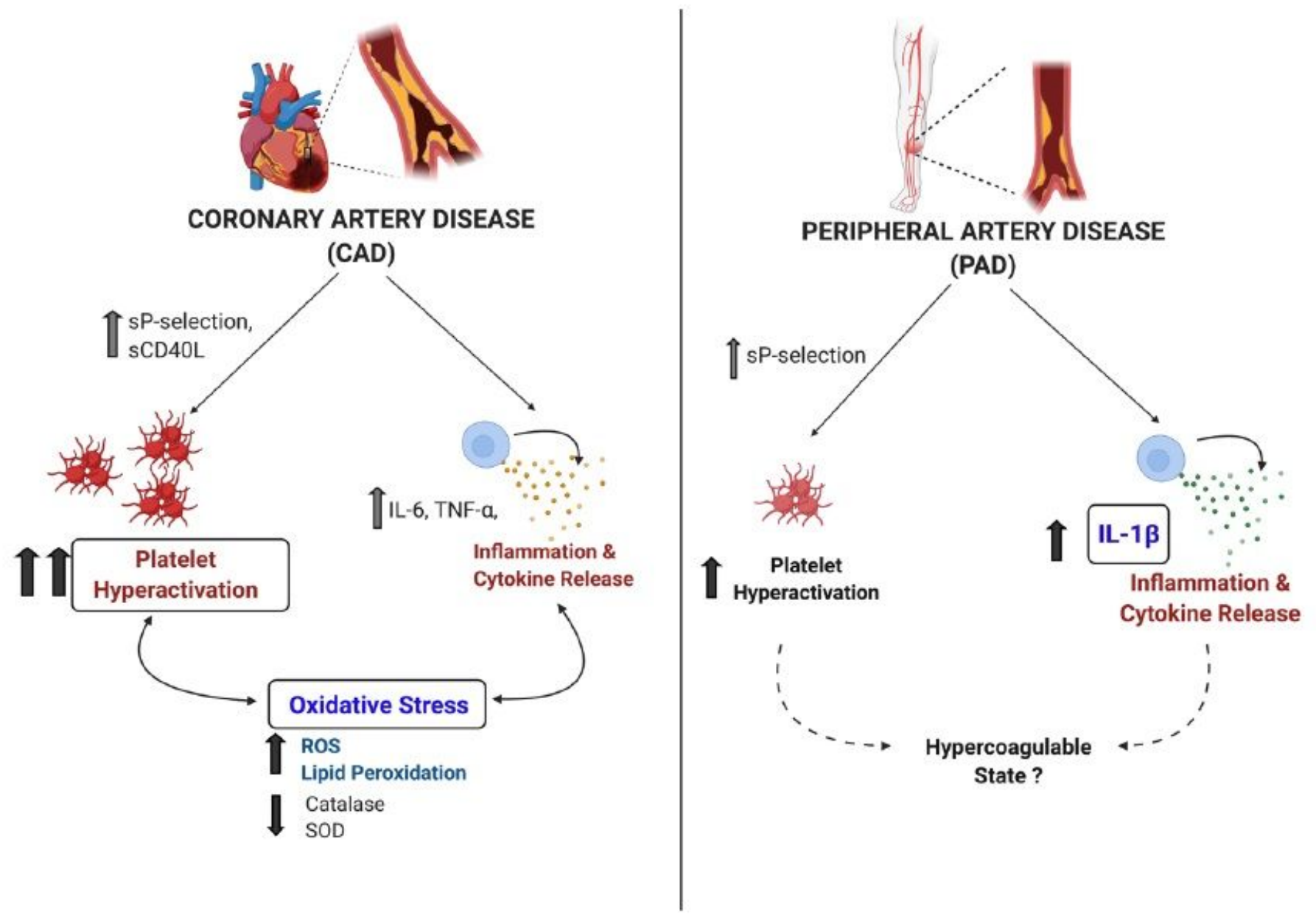

Figure 5

Summary of findings. The two disease processes CAD and PAD are represented diagrammatically. Both platelets and inflammation contribute to the disease processes however there may be differential contribution with platelet activation/ROS playing more of a role in CAD and IL1b-mediated pathology and possible hypercoagulable state playing a prominent role in PAD pathology. This may explain differences in outcomes and response to therapies for CAD and PAD. 